\title{
The effect of xylometazoline spray for expansion of nasal cavity
}

\author{
Jun-Sung Han ${ }^{1}$, Sang-Hi Park ${ }^{1}$, Young Duck Shin ${ }^{1}$, Eunjung Kwon ${ }^{1}$, Woo Sub Shim ${ }^{2}$, and Jin Ho Bae \\ Departments of ${ }^{1}$ Anesthesiology and Pain Medicine, ${ }^{2}$ Otolaryngology-Head and Neck Surgery, College of Medicine, Chungbuk \\ National University, Cheongju, Korea
}

Background: During nasotracheal intubation it is important to have proper pretreatment for nasal mucosa constriction and nasal cavity expanding. Nasal packing of epinephrine gauze is widely used as well as xylometazoline. The aim of this study was to compare and evaluate the efficacy of prophylactic intranasal spray of xylometazoline against epinephrine gauze packing in expanding the nasal cavity.

Methods: Volunteers $(n=32)$ in their twenties without nasal disease such as septal deviation or rhinitis were enrolled in the study. The more patent nostril in each subject was measured by acoustic rhinometry as the base value. After intranasal spray of xylometazoline, the same nostril was remeasured by same method. Twenty four hours later, intranasal packing of epinephrine gauze was done and the same treatment was done. Subject preferences about the procedures were asked.

Results: There were significant difference among treatments (base value: $0.582 \pm 0.164 \mathrm{~cm}^{2}$, xylometazoline spray: $0.793 \pm 0.165 \mathrm{~cm}^{2}$, epinephrine gauze packing: $0.990 \pm 0.290 \mathrm{~cm}^{2}$ ) in acoustic rhinometry. While the epinephrine gauze packing showed more efficient mucosa constriction, subjects preferred xylometazoline spray.

Conclusions: Even though xylometazoline spray was less effective than epinephrine gauze packing, the simplicity and convenience compensated. In patients undergoing nasotracheal intubation, xylometazoline spray can be an alternative to epinephrine gauze packing. (Korean J Anesthesiol 2013; 65: 132-135)

Key Words: Acoustic rhinometry, Epinephrine, Nasal intubation, Xylometazoline.

Received: December 13, 2012. Revised: 1st, January 8, 2013; 2nd, January 24, 2013. Accepted: February 1, 2013.

Corresponding author: Jin Ho Bae, M.D., Department of Anesthesiology and Pain Medicine, College of Medicine, Chungbuk National University, 52, Naesudong-ro, Heungduk-gu, Cheongju 361-763, Korea. Tel: 82-43-269-6233, Fax: 82-43-272-0264, E-mail: jhbae@chungbuk.ac.kr

(c) This is an open-access article distributed under the terms of the Creative Commons Attribution Non-Commercial License (http:// creativecommons.org/licenses/by-nc/3.0/), which permits unrestricted non-commercial use, distribution, and reproduction in any medium, provided the original work is properly cited. 


\section{Introduction}

Airway management is an important consideration when anesthesiologists are doing general anesthesia. Many methods of airway management were invented. For example, nasotracheal intubation is implemented when a patient's mouth does not open well or to assure an operating field when operating on the facial region [1]. However, when an endotracheal tube passes through nasal cavity, it can damage nasal mucosa or cause complications such as nasal bleeding [2]. To reduce these complications, we use many ways to induce constriction of nasal mucosa to expand the nasal cavity and reduce bleeding [3]. Currently, packing gauzes soaked in diluted epinephrine are placed into the nostrils of the patient who is about to be intubated [4]. But if this method is applied before anesthesia induction, it can cause discomfort to patients. If not applied by an expert, it can cause pain. In this study we are evaluating a different method to replace the epinephrine-gauze-packing method. We assess xylometazoline $0.1 \%$ spray (Otrivin $0.1 \%{ }^{\circledR}$, Novartis, Basel, Switzerland), a type of nasal mucosa constrictor.

Xylometazoline $0.1 \%$ spray is a commonly used nasal mucosa constrictor. It is proven to be safe so it can be used without prescription. It can easily cause constriction of nasal mucosa [5]. However, there is not much research compared to the epinephrine-gauze-packing method regarding its effectiveness.

To contrast the two groups, we decided to use acoustic rhinometry (Fig. 1). Because acoustic rhinometry can measure nasal volume conveniently, we thought that technique will help us assess the constriction of nasal mucosa, indirectly but effectively [6].

Before planning this experiment we assumed that if using xylometazoline $0.1 \%$ spray can cause as much nasal mucosa constriction as the epinephrine-gauze-packing method, it would

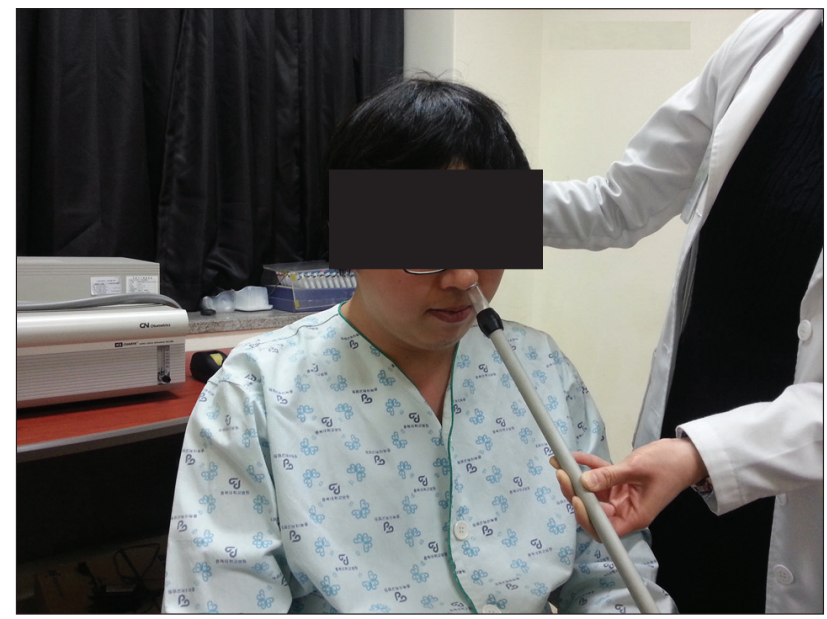

Fig. 1. The acoustic rhinometry shown in action. be useful when doing nasal intubation on real clinic. Also, we decided to compare differences of subjective satisfaction and preferences regarding pain and discomfort with both treatments.

\section{Materials and Methods}

This experiment recruited 32 male volunteers aged 20 to 30 of American Society of Anesthesiologists physical status 1 and 2. The study was approved by the Institutional Review Board and written informed consent was obtained from each volunteer. We excluded volunteers who had nasal septum deviation, allergic rhinitis, who had nasopharyngeal disease history and who showed anatomical variation.

Thirty-two subjects chose the nasal cavity which was most comfortable for them to breathe, and we used only the side they chose for the experiment. We used acoustic rhinometry to measure the cross-section area and volume of chosen nasal cavity. Of the measurements, we chose the value of inferior turbinate, the narrowest section, as a base value (Fig. 2). On the nasal cavity on which the measurements were made, we sprayed xylometazoline $0.1 \%$ spray once. After 5 minutes, using acoustic rhinometry, we measured the cross-section area and volume of the chosen nasal cavity and recorded the value of the narrowest section. More than 24 hours later, when the effect of previous method had worn off, we packed epinephrine gauze in the same nasal cavity. After 5 minutes, we again measured the crosssection area and volume of chosen nasal cavity and recorded the value of the narrowest section. For measurement, we used 3 small-cut $\left(4 \times 8 \mathrm{~cm}^{2}\right)$ gauzes soaked in $1: 100,000$ diluted epinephrine.

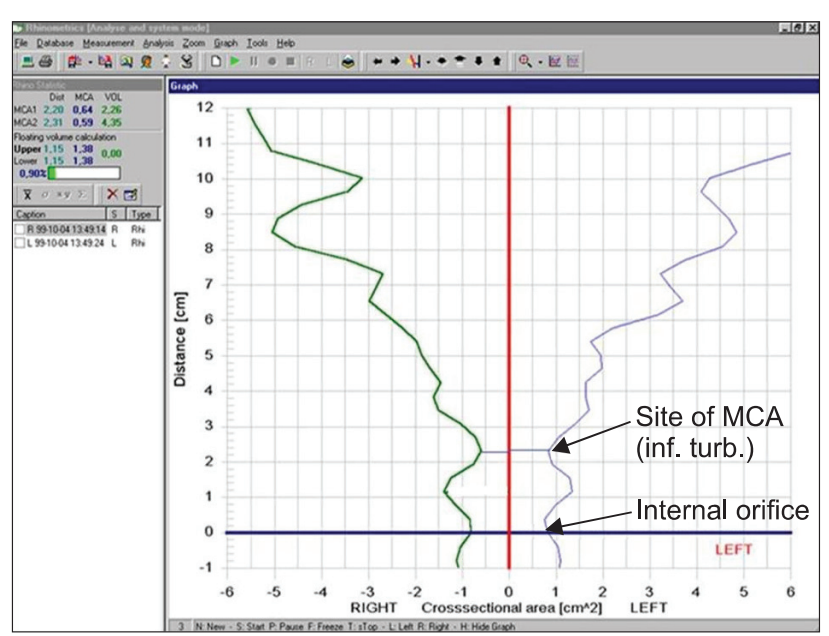

Fig. 2. An example of an acoustic rhinometry trace for one of the study subjects, with nasal cross-sectional area along the $\mathrm{X}$ axis and distance from the wave tube along the $\mathrm{Y}$ axis. MCA: minimum nasal cross-sectional area, inf. turb.: inferior turbinate. 
After the measurement, the volunteers were told to compare subjective feelings when they were treated with xylometazoline $0.1 \%$ spray and when they were treated with epinephrine-gauzepacking method. They were also told to choose which method was more comfortable. Measurement and interviews on every subject were conducted by the same anesthesiologist, within the fixed time.

All results were marked in average and standard deviation. We used repeated measures ANOVA to make comparison on the volume of nasal cavity, and used Chi-square test to test hypothesis of the preference. We rejected null hypotheses of no difference if $P$ values were less than 0.05 . We used SPSS (Version 12.0, SPSS Inc, Chicago, USA).

\section{Results}

Since all 32 volunteers were healthy in their 20's, and the same gender, age $(24.5 \pm 2.1 \mathrm{yr})$, height $(174.5 \pm 6.6 \mathrm{~cm})$, weight $(72.2 \pm 6.7 \mathrm{~kg})$ of the volunteers were similar. For the expansion of the nasal cavity, the reference value was $0.582 \pm 0.164 \mathrm{~cm}^{2}$. After using xylometazoline $0.1 \%$ spray, it was $0.793 \pm 0.165$ $\mathrm{cm}^{2}$. After using the epinephrine-gauze-packing method, it was $0.990 \pm 0.290 \mathrm{~cm}^{2}$. The expanded amount in both cases was significant. It was greatest when using epinephrine-gauzepacking method (Table 1).

On behalf of preference of volunteers, 28 volunteers were in favor of the use of xylometazoline $0.1 \%$ spray and 4 were in favor of epinephrine-gauze-packing method. The difference of preference between two methods was significant.

\section{Discussion}

Tracheal intubation is the most basic and most important skill for anesthesiologists. For this reason, various methods of tracheal intubation exist. Among them, nasotracheal intubation is one method which is not difficult to use, with broad applications. Nasotracheal intubation can be used during operation on oral cavity, nasopharynx, face etc. It can also be used in situations when long-term intubation is needed, such as motor disturbance of mandible fracture or temporomandibular joints,

Table 1. Comparison of Nasal Cavity Area

\begin{tabular}{cccc}
\hline & Base values & $\begin{array}{c}\text { Xylometazoline } \\
\text { spray }\end{array}$ & $\begin{array}{c}\text { Epinephrine } \\
\text { packing }\end{array}$ \\
\hline Nasal cavity $\left(\mathrm{cm}^{2}\right)$ & $0.582 \pm 0.164^{*}$ & $0.793 \pm 0.165^{\dagger}$ & $0.990 \pm 0.290^{\ddagger}$ \\
\hline
\end{tabular}

Values are mean \pm SD. Base values: The area of nasal cavity without pretreatment. Xylometazoline spray: The area of nasal cavity after xylometazoline spray. Epinephrine packing: The area of nasal cavity after epinephrine gauze packing. ${ }^{*, \dagger}$, There were significant differences among the three groups $(\mathrm{P}<0.001)$. obstacle existing on oral cavity or oropharyngeal. However, there can be many complications when using nasotracheal intubation. Kim et al. [7] stated that when using nasotracheal intubation, there was $27.1 \%$ chance of complications. For example, there could be nasal bleeding, damage on nasal mucosa or on nasopharyngeal mucosa, nasal polyp, or bleeding and airway obstruction caused by the movement of nasal foreign body. Nasal bleeding is the most common complication [4].

To prevent nasal bleeding caused by nasotracheal intubation, many methods are used. Among them are using a small tube, doing intubation by tube warming or fiberoptic bronchoscopy, using lubricating gel and nasal mucosa constrictor, and packing diluted epinephrine-soaked gauze before intubation $[3,4]$. But if this method is applied before anesthesia induction, the packing itself can cause discomfort, and if not applied by an expert, can inflict pain during the treatment.

Xylometazoline spray is a kind of nasal mucosa constrictor and it is $\alpha-1$ agonist. It is a non prescription drug, meaning its safety is confirmed. It can be easily used to expand the nasal cavity $[5,8]$. However there are very few studies of comparisons with epinephrine-gauze-packing method. If we can prove xylometazoline spray treatment has an effect similar to epinephrine-gauze-packing method, it can be said that spray treatment can replace epinephrine-gauze-packing method. This is why we devised this study. In other studies, there were many cases when researchers spayed xylometazoline spray multiple times $[5,8]$. However, in this study we only sprayed only once as the drug production company recommended, because the subjects were volunteers, not patients.

El-Seify et al. [8] stated that among children who had nasotracheal intubation, the occurrence of nasal bleeding for the group that was pretreated with xylometazoline spray was significantly less compared to the group that was treated only with physiological saline. However the comparison of the effect was not with the epinephrine-gauze-packing method. In other literature as well there is little research on the comparison with epinephrine-gauze-packing method. Moreover, because previous research methods made the occurrence of nasal bleeding a result index after pretreatment, the research itself is difficult to achieve. Also, in hospitals where nasotracheal intubation is not implemented frequently, it is difficult to do the research. Therefore, in this research, to compare the difference, we decided to use acoustic rhinometry, which is simple and noninvasive.

Acoustic rhinometry is a test method that objectively measures blockage of nasal cavity following changes of nasal cavity. Acoustic rhinometry shoots sounds of various frequencies inside nasal cavity and analyzes the returning signal. With simple cooperation of examinee it can measure cross-section area and volume of nasal cavity of random parts in a very short time. Acoustic rhinometry is effectively used in otolaryngology 
mainly when comparing changes after using mucous constrictor or operating nasal cavity operation on patients who have blocked nasal cavity $[9,10]$. We noticed that the purpose of both epinephrine-gauze-packing and xylometazoline spray is to expand the volume of nasal cavity and to prevent nasal bleeding through constriction of mucous. Because comparison of the difference of the volume of nasal cavity made by acoustic rhinometry can evaluate the degree of constriction of nasal cavity, and furthermore can be used as an index to predict the occurrence of nasal bleeding depending on the degree of constriction, we made it an evaluation index in this study. The outer diameter of the wire-reinforced tube (internal diameter is $6.5 \mathrm{~mm}$ ) that is frequently used during nasotracheal intubation of adult male patients is $8.9 \mathrm{~mm}$, and the external area of it is about $0.62 \mathrm{~cm}^{2}$. The narrowest area of nasal cavity was $0.582 \pm 0.164 \mathrm{~cm}^{2}$ before any treatment was done. After using xylometazoline spray, it was $0.793 \pm 0.165 \mathrm{~cm}^{2}$, and after using epinephrine-gauze-packing method, it was $0.990 \pm 0.290 \mathrm{~cm}^{2}$. Compared to the area of external diameter of the tube, we can assume that both methods have shown significant increase. Even though we cannot conclude whether the expansion of nasal cavity helps reduce nasal bleeding because of lack of research, we still can assume that it can be used as a useful device. We also think that pre-anesthetic evaluation through acoustic rhinometry is simpler and more objective than existing post-anesthetic nasal bleeding check test.
In this research, we could see that both methods were effective on expanding the area of nasal cavity since measurement made by acoustic rhinometry differed significantly compared to the base value. However, after doing epinephrine-gauze-packing there was the larger expansion of nasal cavity, significantly larger than xylometazoline spray. Therefore, based on this result we can conclude that epinephrine-gauze-packing was the most effective method. Using xylometazoline spray was also proven effective, but its effect was less.

Regarding volunteer preferences, 28 out of 32 chose xylometazoline spray. Before the experiment, we predicted that all people would prefer xylometazoline spray. However, 4 volunteers preferred epinephrine-gauze-packing because they didn't like the feeling when xylometazoline spray was sprayed.

From these results, we can conclude that even though xylometazoline spray has some effect on the expansion of nasal cavity and helps reduce nasal bleeding, its effects are not that as effective as those of epinephrine-gauze-packing. However, we think that convenience for patients and doctors can make up for this insufficiency in some degree. It can be employed usefully for patients who are getting a nasotracheal intubation.

\section{Acknowledgments}

This work was supported by the research grant of the Chungbuk National University in 2011.

\section{References}

1. Henderson J. Airway management in the adult. In: Miller's Anesthesia. 7th ed. Edited by Miller RD: Philadelphia, Churchill Livingstone. 2009, pp 1586-7.

2. Kim HJ, Yum KW. A Prospective study on complications after nasotracheal intubation in oral and maxillofacial surgery patients. Korean J Anesthesiol 2000; 39: 72-6.

3. Enk D, Palmes AM, Van Aken H, Westphal M. Nasotracheal Intubation: a simple and effective technique to reduce nasopharyngeal trauma and tube contamination. Anesth Analg 2002; 95: 1432-6.

4. Yoo RH, Han YJ, Jin YH, Song HS. Effects of nasopharyngeal airway on incidence of epistaxis and ease of tube advancement during nasotracheal intubation. Korean J Anesthesiol 1999; 36: 955-9.

5. Eskiizmir G, Hirçin Z, Ozyurt B, Unlü H. A comparative analysis of the decongestive effect of oxymetazoline and xylometazoline in healthy subjects. Eur J Clin Pharmacol 2011; 67: 19-23.

6. Bae SH, Mo JH, Chung YJ. Correlation between improvement of subjective nasal patency and changes of parameters measured by acoustic rhinometry after septoplasty and outfracturing of both turbinates. Korean J Otorhinolaryngol-Head Neck Surg 2011; 54: 131-6.

7. Kim MH, Son JS, Lim HS, Kim DK, Kim DC. Effective removal of epistaxis during nasotracheal intubation utilizing a fiberoptic scope in a difficult airway - a case report -. Korean J Anesthesiol 2008; 55: 618-20.

8. El-Seify ZA, Khattab AM, Shaaban AA, Metwalli OS, Hassan HE, Ajjoub LF. Xylometazoline pretreatment reduces nasotracheal intubationrelated epistaxis in paediatric dental surgery. Br J Anaesth 2010; 105: 501-5.

9. Cakmak O, Coskun M, Celik H, Büyüklü F, Ozlüoglu LN. Value of acoustic rhinometry for measuring nasal valve area. Laryngoscope 2003; 113: 295-302.

10. Fisher EW, Lund VJ, Scadding GK. Acoustic rhinometry in rhinological practice: discussion paper. J R Soc Med 1994; 87: 411-3. 